\title{
A Fast Technique for Motion Correction in DAS Using a Feature-Based, Irregular Grid ${ }^{\star}$
}

\author{
Erik H.W. Meijering, Karel J. Zuiderveld, Max A. Viergever \\ Image Sciences Institute, Utrecht University, Heidelberglaan 100, 3584 CX Utrecht, \\ The Netherlands. http://www.isi.uu.nl; e-mail: erik@isi.uu.nl.
}

\begin{abstract}
In clinical practice, Digital Subtraction Angiography (DSA) is a powerful technique for the visualization of blood vessels in the human body. However, due to patient motion the diagnostic relevance of the images is often reduced by the introduction of artifacts. In this paper, we propose a new approach to the registration of DSA images, which is both effective, and very fast. The computational speed of our algorithm is achieved by applying a gradient based control point selection mechanism, which allows for a more efficient positioning of a reduced number of control points as compared to approaches based on regular grids. The results of preliminary experiments with several clinical data sets clearly show the applicability of the algorithm.
\end{abstract}

\section{Introduction}

In clinical practice, Digital Subtraction Angiography (DSA) is a powerful technique for the visualization of blood vessels in the human body. With this technique, a sequence of X-ray projection images is taken to show the passage of a bolus of injected contrast material through one or more vessels of interest. The background structures in these contrast images are largely removed by subtracting an image taken prior to the arrival of the contrast medium: the mask image. The subtraction technique is based on the assumption that during exposure, tissues do not change in position or density. Clinical evaluations following the introduction of DSA in the early eighties $[1,2]$ revealed that this assumption is not valid for a substantial number of examinations. Patient motion frequently occurs, which causes the subtraction images to show artifacts that may hamper proper diagnosis. In order to reduce motion artifacts, the misalignment of the successive images in the sequence needs to be determined and corrected for; operations often referred to as registration and motion correction $[3,4]$.

Although many studies have been carried out on this subject over the past two decades, they have not led to algorithms which are sufficiently fast so as to be acceptable for integration in a clinical setting. In this paper, we propose a new approach to the registration of digital angiographic image sequences which

* This work was done in cooperation with Philips Medical Systems, Department of $\mathrm{X}$-Ray Diagnostics/Predevelopment, Best, The Netherlands and was supported by the Netherlands Ministry of Economic Affairs (IOP-project IBV96004). 
is both effective, and computationally very efficient. The registration approach is described in Section 2. Results of preliminary experiments showing the performance of the algorithm are presented in Section 3, and discussed in Section 4. Concluding remarks are made in Section 5 .

\section{Registration Approach}

Given a two-dimensional digital image sequence $I(x, y, t)$ of size $M \times M \times N$, the registration of one of the images with respect to a successive image in the sequence, involves two operations: (i) The computation of the correspondence between the pixels in the two images; (ii) The correction based on this correspondence. In this section, the proposed approach to perform these operations is described.

\subsection{Control Point Selection}

It is computationally very expensive to compute the correspondence separately for every pixel. To reduce the computation time to a clinically acceptable level (several seconds), assumptions have to be made concerning the nature of the underlying motion. From a physical point of view it is reasonable to assume a certain amount of coherence between the motion of neighboring pixels and to compute the correspondence only for a selected amount of control points $\mathbf{p}_{i}=\left(x_{i}, y_{i}\right)$. The overall correspondence can then be obtained by interpolation. Control points can be chosen manually by selecting a region of interest [5], or can be taken to lie on a regular grid $[6-8]$. More sophisticated algorithms use image features.

In the subtraction images, artifacts can only appear in those regions where strong object edges are present in the individual images. Moreover, edges can be matched better than more homogeneous regions. Therefore, the selection of control points should be based on an edge-detection scheme. Compared to algorithms where control points are chosen on a regular grid, this has three major advantages: (i) The control points are chosen at those positions where the artifacts can be expected to be largest; (ii) The reliability of the displacement estimates will be higher than in the case of arbitrarily selected points; (iii) The number of control points, and thus computation time, is reduced.

The location of edges can easily be computed by detecting local maxima of the grey-level gradient magnitude $\|\nabla I(x, y)\|$ of the mask image. In our algorithm, $\|\nabla I(x, y)\|$ is computed at a relatively small scale $\sigma$, using Gaussian regularization [9]. Since, in principle, the potential severeness of a motion artifact is directly related to the strength of the underlying edge, it is required to indicate which of the edges are sufficiently important to be considered further. The final selection of control points from $\|\nabla I(x, y)\|$ is carried out by dividing the image into blocks of size $D_{\max } \times D_{\max }$. In turn, these blocks are subdivided into smaller blocks of size $D_{\min } \times D_{\min }$. For every large block, every interior 

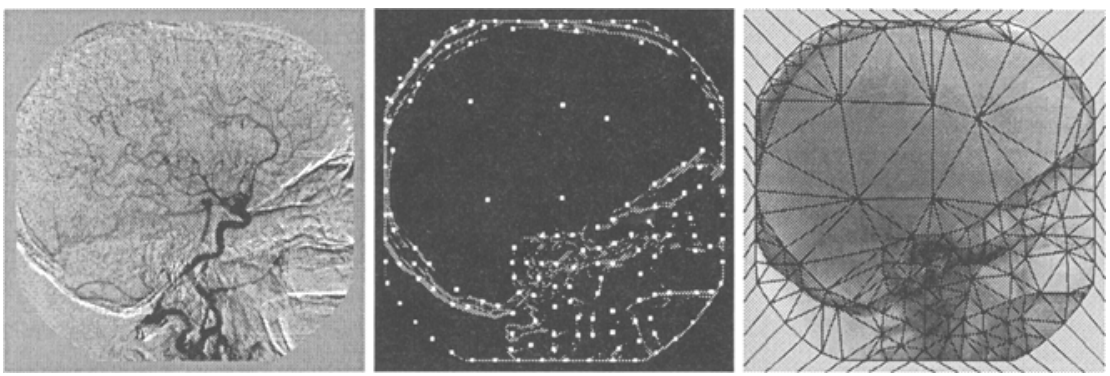

Fig. 1. An example of control point selection and mesh generation. Left: A cerebral DSA image showing motion artifacts. Middle: The thresholded gradient magnitude image of the mask, used as a prediction of the locations of motion artifacts, with the set of selected control points (white dots) superimposed. Right: The mask image with the resulting mesh (Delaunay triangulation) superimposed.

small block is scanned for pixels at which $\|\nabla I(x, y)\|$ is above a certain threshold $\Theta_{e}$. From these pixels, the one with the largest value for $\|\nabla I(x, y)\|$ is taken as a candidate control point. If no edge-pixels are encountered, no candidate is selected. The candidate becomes a control point if it is positioned inside the exposure region $\mathcal{R}_{E}$ and at a distance of at least $D_{\text {exp }}$ from the border $\partial \mathcal{R}_{E}$ of that region ${ }^{1}$. In order to enforce a minimum distance between control points, the gradient magnitude values in a $\left(2 D_{\min }+1\right) \times\left(2 D_{\min }+1\right)$ region around the selected point (that point being the center) are suppressed. If no point is selected after a large block has been scanned, the point with the largest gradient magnitude value in a small block around the center of the large block is taken as a control point so as to constrain the maximum distance between selected points.

\subsection{Displacement Computation}

For the computation of the actual displacements of the selected control points we use a template matching approach: for every control point, a small window $\mathcal{W}$ of $W \times W$ pixels is defined and the corresponding window in a successive image in the sequence is searched for by optimizing a predefined measure of match. In order for such a technique to be successful in angiographic images, the similarity measure is required to be insensitive to the inflow of contrast in some parts of the image. It has been demonstrated by Buzug et al. $[10,11]$ that measures based on the histogram of difference are most suitable for this purpose. In order to find the optimal displacement in an efficient manner, we use a hill-climbing optimization approach.

${ }^{1}$ By $\mathcal{R}_{E}$ we denote the part of the image in which the grey-values were caused by the exposure of X-rays. Because of the shape of the image intensifier, it is more or less circular (see e.g. Fig. 1) and can be extracted quite easily. 
As indicated by a clinical evaluation in the early eighties [2], even subpixel misalignments may produce significant artifacts in the subtraction image. In our algorithm, subpixel accuracy in the displacement computations is accomplished by local interpolation of the images and by determining the optimal measure of match at subpixel displacements around the optimum of integer displacements. An accuracy of 0.1 pixel is sufficient for angiographic images [7].

\subsection{Displacement Interpolation}

In order to be able to carry out the warping of the mask image with respect to the contrast image, it is required to have a complete description of the displacement vector field $\mathbf{d}: \mathbb{R}^{2} \rightarrow \mathbb{R}^{2}$. In order to reduce the computation time to a minimum, the displacements at the remaining points in the image are linearly interpolated from the displacements at the control points. Therefore it is required that the set $P=\left\{\mathbf{p}_{i}\right\}$ of control points is properly tessellated. The only possible polygons that can be used for this purpose are triangles, so that the control points become the vertices of an irregular triangular mesh.

In our algorithm, the control points are tessellated into a Delaunay triangulation $\mathcal{D}(P)[12]$, since in this case it is guaranteed that the smallest of the three angles of every triangle will be as large as possible, thereby avoiding triangles with one or two highly acute interior vertex angles in which the displacement at certain points will become dependent on very distant control points (see Fig. 1). An important advantage of using such a tessellation is that the required operations for the final warping of the polygons can be carried out by graphics hardware, which will result in a very fast implementation.

\section{$3 \quad$ Experimental Results}

The algorithm as presented in the previous section was implemented in the $\mathrm{C}++$ programming language, utilizing the Open Graphics Library. The experiments were carried out on a relatively low cost Silicon Graphics O2 workstation (one 180 MHz R5000 IP32 processor, $64 \mathrm{MB} / 512 \mathrm{kB}$ main/cache memory), with graphics hardware for support of the OpenGL instructions. During the experiments, the parameters of the algorithm as described in the previous section were kept fixed at the values shown in Table 1. Clinical digital angiographic image sequences were acquired on a Philips Integris system. For all sequences, the first image was taken as the mask image.

The results of applying the proposed registration technique to a cerebral, peripheral and an abdominal image sequence are shown in Fig. 2. The left column shows the original subtraction images which contain motion artifacts. The middle column shows the registration results after applying a manual pixel-shifting technique ${ }^{2}$, as provided on standard DSA imaging devices. With this technique, the regions for which corrections must be obtained have to be selected explicitly:

\footnotetext{
${ }^{2}$ That is, global translation only.
} 


\begin{tabular}{|c|c|c|c|}
\hline $\mathrm{Pa}$ & Value & Param. & Value \\
\hline$\sigma$ & 1.0 & $D_{\min }$ & $0.04 M$ \\
\hline$\theta_{e}$ & 15.0 & $D_{\max }$ & $0.20 M$ \\
\hline$W$ & 51 & $D_{\text {exp }}$ & $0.01 M$ \\
\hline
\end{tabular}

Table 1. The values of the parameters of the algorithm during the experiments. $M$ denotes the $x$ - $y$-dimension of the image sequence (either 1024 or 512 pixels in most DSA sequences). See further Section 2 for a description of these parameters.

In the cerebral image we corrected for artifacts in the middle-right part of the image; In the peripheral image, artifacts were corrected in the left part of the image; Finally, in the abdominal image, we attempted to correct for artifacts in the top-left part. Although some artifacts were eliminated by this procedure, in other parts of the images artifacts were reinforced or even introduced. That is to say, the artifacts could not be corrected for by means of $2 \mathrm{D}$ rigid transformations, even though the original 3D motion might have been rigid, e.g. in the case of the cerebral image. In general, the approach proposed in the present paper, based on a triangular mesh of irregularly spaced, edge-based control points yields better registrations and, consequently, better subtractions.

\section{Discussion}

It must be pointed out that angiographic images are in fact two-dimensional representations of a three-dimensional scene. It has been shown by Fitzpatrick [13] that, subject to a few conditions (which are easily met in X-ray projection imaging), given any two such images that are assumed to differ only as a result of the motion of particles in the three-dimensional scene, there does exist a one-to-one two-dimensional mapping that completely describes the changes in the images, caused by three-dimensional object motion. However, it should be stressed that it is not possible to uniquely and completely retrieve it from the two images only. There are three main reasons for this: (i) Since we are dealing with digital images, the retrieval of a displacement vector in a certain point in one image in a sequence inevitably requires neighborhood operations. If the neighborhood contains several objects that move independently of each other (which is very likely to occur in projection images), the computations can be expected to be unreliable and inaccurate; (ii) At points that lie on isophotes in the image, it is impossible to retrieve the tangential component of the displacement vector (generally known as the aperture problem); (iii) The assumption that the two images differ only as a result of the motion of particles in the three-dimensional scene, does not apply to angiography, since contrast material is introduced in certain parts of the scene. Although similarity measures can be designed to be relatively insensitive to inflow of contrast, the problems (i) and (ii) are more fundamental and cannot be solved without additional knowledge. 

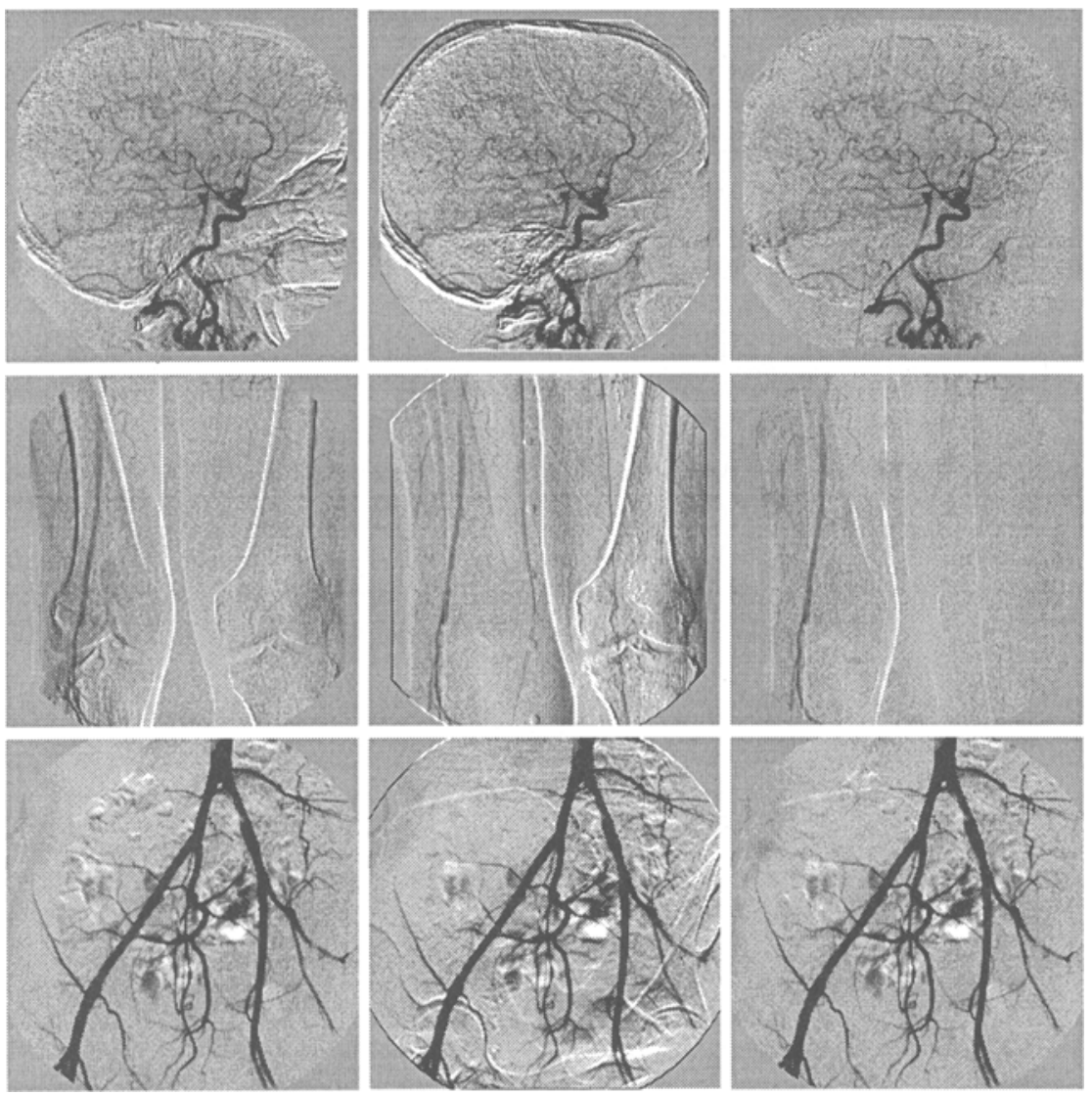

Fig. 2. Registration results for a cerebral, peripheral and an abdominal image sequence. Left Column: The original subtractions of one of the contrast images from the mask image. Middle Column: The subtractions after registration by global translation of the mask image (obtained by manual pixel-shifting; see Section 3 for a more detailed explanation). Right Column: The subtractions after registration using the proposed approach.

Therefore, successful application of our registration technique will be limited to regions where these problems are insignificant.

From the experimental results as shown in Fig. 2, it can be seen that there is a major difference between the registration results of the cerebral and peripheral sequences and the results of the abdominal sequence. In the first two sequences, the artifacts have been removed almost completely, i.e., the algorithm gave near perfect registrations. In the abdominal data set, the motion artifacts have been removed partially, but the resulting subtraction still shows some major artifacts. We note that these artifacts could not be removed by adjusting one or more of the parameters of the algorithm so as to yield a larger density of control 
points. In fact, the artifacts could not even be removed by replacing the hillclimbing optimization by an exhaustive-search approach. The registration result of the abdominal sequence where manual pixel-shifting was applied reveals that there are parts in the image in which there are several important structures projected onto each other (in this case the spine, the pelvis and the bowels), as opposed to the cerebral and peripheral sequences, where the important structures resulted from the projection of only one object in the original three-dimensional scene, viz. bones. When multiple superimposed structures move independently, the displacement of only one of these structures can no longer be reconstructed from the resulting projections. This has been mentioned at the beginning of this section as the first limitation of any registration algorithm for X-ray projection images, and explains why even a full-search approach will not be able to retrieve the correspondence. In general, it was found that in most abdominal data sets the peristaltic motion locally causes severe changes in grey-values. In those cases, even when the correct local displacements are found, the final subtraction will still show serious artifacts. It is inherent to the nature of the current approach that these artifacts cannot be removed.

Finally, we note that the proposed approach is capable of registering at a speed of less than one second per image (on average). This is mainly due to the edge-based control point selection procedure which, in general, results in a reduction in the number of points for which the displacement needs to be computed explicitly.

\section{Conclusions}

We have presented a new approach for the registration of digital angiographic image sequences. The method involves the extraction of regions in the image where artifacts can be expected to appear in the case of patient motion. These regions are obtained by thresholding the gradient magnitude of the mask image. Based on assumptions about the coherence of motion of neighboring pixels, a selected set of control points is extracted, for which the displacement is computed explicitly by means of maximizing the energy of the histogram-of-differences and using a hill-climbing optimization approach. The complete displacement vector field is constructed from the displacements at these control points, using a Delaunay triangulation and linear interpolation. The final warping of the images is performed real-time by graphics hardware.

The algorithm is capable of registering complete sequences at a speed of less than one second per image. The results of a preliminary evaluation on various clinical image sequences show the viability of the method. We are currently undertaking a thorough clinical evaluation of the algorithm.

\section{References}

1. W.A. Chilcote, M.T. Modic, W.A. Pavlicek, J.R. Little, A.J. Furian, P.M. Duchesneau \& M.A. Weinstein. Digital subtraction angiography of the carotid arteries: A comparitive study in 100 patients. Radiology, vol. 139, no. 2 (1981), pp. 287-295. 
2. W.R. Brody, D.R. Enzmann, L.-S. Deutsch, A. Hall \& N. Pelc. Intravenous carotid arteriography using line-scanned digital radiography. Radiology, vol. 139, no. 2 (1981), pp. 297-300.

3. L.G. Brown. A survey of image registration techniques. ACM Computing Surveys, vol. 24 , no. 4 (1992), pp. 325-376.

4. J.B.A. Maintz \& M.A. Viergever. A survey of medical image registration. Medical Image Analysis, vol. 2, no. 1 (1998), pp. 1-36.

5. A. Venot \& V. Leclerc. Automated correction of patient motion and gray values prior to subtraction in digitized angiography. IEEE Transactions on Medical Imaging, vol. 3, no. 4 (1984), pp. 179-186.

6. K.J. Zuiderveld, B.M. ter Haar Romeny \& M.A. Vjergever. Fast rubber sheet masking for digital subtraction angiography. In Science and Engineering of Medical Imaging, M.A. Viergever (ed.), vol. 1137 of Proceedings of SPIE, The International Society for Optical Engineering, Bellingham, Washington, USA, 1989, pp. 22-30.

7. L. van Tran \& J. Sklansky. Flexible mask subtraction for digital angiography. IEEE Transactions on Medical Imaging, vol. 11, no. 3 (1992), pp. 407-415.

8. G.S. Cox \& G. de Jager. Automatic registration of temporal image pairs for digital subtraction angiography. In Image Processing, vol. 2167 of Proceedings of SPIE, The International Society for Optical Engineering, Bellingham, Washington, USA, 1994, pp. 188-199.

9. J.F. Canny. A computational approach to edge detection. IEEE Transactions on Pattern Analysis and Machine Intelligence, vol. 8, no. 6 (1986), pp. 679-698.

10. T.M. Buzug, J. Weese, C. Fassnacht \& C. Lorenz. Image registration: Convex weighting functions for histogram-based similarity measures. In CVRMed-MRCAS '97, J. Troccaz, E. Grimson \& R. Mösges (eds.), vol. 1205 of Lecture Notes in Computer Science, Springer-Verlag, Berlin, Germany, 1997, pp. 203-212.

11. T.M. Buzug, J. Weese, C. Lorenz \& W. Beil. Histogram-based image registration for digital subtraction angiography. In Image Analysis and Processing (ICIAP '97), A. Del Bimbo (ed.), vol. 1311 of Lecture Notes in Computer Science, SpringerVerlag, Berlin, Germany, 1997, pp. 380-387.

12. D.F. Watson. Computing the $n$-dimensional Delaunay tessellation with application to Voronoi polytopes. The Computer Journal, vol. 24, no. 2 (1981), pp. 167-172.

13. J.M. Fitzpatrick. The existence of geometrical density-image transformations corresponding to object motion. Computer Vision, Graphics and Image Processing, vol. 44, no. 2 (1988), pp. 155-174. 